\title{
ALGODOEIRO CULTIVADO EM SOLO TRATADO COM CALCÁRIO, LODO DE ESGOTO E CÁDMIO
}

\author{
Thiago Assis Rodrigues Nogueira ${ }^{1,2}$, Marcos Omir Marques ${ }^{1}$, \\ Fernando Almeida Muçouçah ${ }^{1}$, Ivana Machado Fonseca ${ }^{1}$
}

${ }^{1}$ Universidade Estadual Paulista, Faculdade de Ciências Agrárias e Veterinárias. Via de acesso Prof. Paulo Donato Castellane, s/n. CEP. 14.884-900 - Jaboticabal, São Paulo,

Brasil. ${ }^{2}$ Bolsista Fapesp. Correspondência: tarnogueira@ click21.com.br

\section{Cotton plants cultivated in soil amended with lime, sewage sludge and cadmium}

Key words: Gossypium hirsutum, biosolid, liming, trace elements, organic residue.

\begin{abstract}
The objective of the work was to evaluate the accumulation of nutrients and heavy metals in different parts of the cotton plants (Gossypium hirsutum cv. IAC 22) cultivated in soil that received lime and sewage sludge from municipal treatment plant plus cadmium. The experiment was carried out under green house conditions. The soil was a Clayey Red Latosol (Oxisol). The experimental design was the randomized blocks. The treatments were organized in a $3 \times 2+1$ factorial scheme (three lime rates, two sewage sludge rates and a Control treatment), all with three replications. The variance test was made by $\mathrm{F}$ test and means comparisons by Tukey test $(\mathrm{p}<0.05)$. The different lime rates provided larger macronutrientes accumulations in the shoot and micronutrients in the roots. The sewage sludge addition provided the macronutrients accumulation, especially, in the roots and shoot of the plants, and of $\mathrm{Mn}$ and $\mathrm{Zn}$ in the root and shoot and just $\mathrm{Mn}$ in the fibers. The Fe accumulation in the roots decreased with the application of the sewage sludge. The liming and the sewage sludge provided accumulation of $\mathrm{Pb}$ in the roots, shoot and fibers. When it was just considered the liming, similar effect was found for the $\mathrm{Cd}$ and $\mathrm{Ni}$ in the fibers.
\end{abstract}

Palavras-chave: Gossypium hirsutum, biossólido, calagem, elementos traço, resíduo orgânico 


\section{RESUMO}

O objetivo do trabalho foi avaliar o acúmulo de nutrientes e metais pesados em diferentes partes das plantas de algodoeiro (Gossypium hirsutum cv. IAC 22) cultivadas em solo que recebeu calcário e lodo de esgoto contaminado com cádmio. $\mathrm{O}$ experimento foi conduzido em casa de vegetação na FCAV/Unesp, em Jaboticabal-SP, em Latossolo Vermelho argiloso. Utilizou-se o delineamento experimental em blocos casualizados em esquema fatorial 3 x $2+1$, sendo três doses de calcário calcinado, duas doses de lodo de esgoto e uma testemunha adicional, com três repetições. Procedeu-se à análise de variância e ao teste Tukey a $5 \%$ de probabilidade. As diferentes doses de calcário proporcionaram maiores acúmulos de macronutrientes na parte aérea e micronutrientes nas raízes. A adição de lodo proporcionou o acúmulo de macronutrientes, especialmente, nas raízes e parte aérea das plantas, e de Mn e Zn na raiz e parte aérea e apenas Mn nas fibras. O acúmulo de Fe nas raízes diminuíram com a aplicação do lodo de esgoto. A calagem e o lodo proporcionaram acúmulo de $\mathrm{Pb}$ nas raízes, parte aérea e fibras. Quando se considerou apenas a calagem, efeito semelhante foi encontrado para o $\mathrm{Cd}$ e Ni nas fibras.

\section{INTRODUÇÃO}

O aumento da população dos centros urbanos é o principal responsável pela produção de diversos resíduos, que podem ser acumulados no ambiente sem o adequado tratamento ou utilização que possibilite sua reciclagem. Dentre os diferentes tipos encontrados, podese destacar o lodo de esgoto, que é um resíduo de composição predominantemente orgânica, obtido ao final do tratamento de águas servidas. Por ser gerado em larga escala, é fonte constante de preocupação no que se refere à contaminação ambiental (Rocha et al., 2003).

A presença de matéria orgânica e de nutrientes de plantas na composição do lodo de esgoto sanitário lhe confere potencial para ser utilizado como fertilizante e condicionador do solo, podendo substituir, ainda que parcialmente, os fertilizantes minerais (Nascimento et al., 2004).

A reciclagem agrícola do lodo de esgoto destaca-se por reduzir a pressão sobre a exploração dos recursos naturais para a produção de fertilizantes e os custos decorrentes dos insumos agrícolas nos sistemas produtivos. Além disso, diminui o impacto ambiental causado na medida em que dispensa a adoção de outras opções de destino.

A reciclagem de nutrientes no solo, com o aproveitamento agrícola do lodo de esgoto, apresenta-se como tendência mundial (Lopes et al., 2005). Entretanto, devido à possibilidade de presença de metais pesados, sua adição continuada no solo deve ser avaliada de forma criteriosa, considerandose os riscos de contaminação que representa e sua eventual transferência à cadeia alimentar (Nriagu e Pacyna, 1988; Mesquita, 2002).

Dentre as culturas com possibilidades de receber o lodo de esgoto, destaca-se o algodoeiro. Sua importância decorre da fibra que compõe o algodão, matéria prima de grande importância para a indústria têxtil, ao mesmo tempo em que não representa risco à saúde humana (Pedroza et al., 2003).

$\mathrm{O}$ presente trabalho teve como objetivo avaliar o acúmulo de macronutrientes $(\mathrm{N}$, $\mathrm{P}, \mathrm{K}, \mathrm{Ca}, \mathrm{Mg}$ e S), micronutrientes (Fe, $\mathrm{Mn}$ e $\mathrm{Zn}$ ) e metais pesados ( $\mathrm{Cd}$, Ni e $\mathrm{Pb}$ ) nas diferentes partes de plantas de algodoeiro cultivadas em solo acrescido de lodo de esgoto contaminado, de forma intencional, com cádmio.

\section{MATERIAL E MÉTODOS}

O experimento foi conduzido em condições de casa de vegetação, nas dependências do Departamento de Tecnologia da FCAVUNESP, Campus de Jaboticabal, SP. O solo 
Quadro 1: Propriedades químicas do LATOSSOLO VERMELHO argiloso. Table 1: Clayey Red Latosol (Oxisol) chemical properties.

\begin{tabular}{lcc}
\hline Propriedade & Valor & DESVPAD $^{1}$ \\
\hline $\mathrm{pH}\left(\mathrm{CaCl}_{2}\right)$ & 4,2 & 0,25 \\
Matéria Orgânica $\left(\mathrm{g} \mathrm{dm}^{-3}\right)$ & 9,0 & 0,36 \\
$\mathrm{P}-\mathrm{resina}\left(\mathrm{mg} \mathrm{dm}^{-3}\right)$ & 7,0 & 0,26 \\
$\mathrm{~K}\left(\mathrm{mmol}_{\mathrm{c}} \mathrm{dm}^{-3}\right)$ & 1,0 & 0,26 \\
$\mathrm{Ca}\left(\mathrm{mmol}_{\mathrm{c}} \mathrm{dm}^{-3}\right)$ & 4,0 & 0,26 \\
$\mathrm{Mg}\left(\mathrm{mmol}_{\mathrm{c}} \mathrm{dm}^{-3}\right)$ & 2,0 & 0,17 \\
$\mathrm{H}+\mathrm{Al}_{\left(\mathrm{mmol}_{\mathrm{c}} \mathrm{dm}^{-3}\right)}$ & 34,0 & 3,46 \\
$\mathrm{SB}\left(\mathrm{mmol}_{\mathrm{c}} \mathrm{dm}^{-3}\right)$ & 7,0 & 0,26 \\
$\mathrm{~T}\left(\mathrm{mmol}_{\mathrm{c}} \mathrm{dm}^{-3}\right)$ & 41,0 & 3,56 \\
$\mathrm{~V}(\%)$ & 17,0 & 1,43 \\
\hline
\end{tabular}

${ }^{1}$ Desvio Padrão.

empregado foi um Latossolo Vermelho argiloso (Embrapa, 1999), com características químicas (Quadro 1) determinadas de acordo com Raij et al. (1987).

Foi utilizando como planta-teste o algodoeiro (Gossypium hirsutum L.) cultivar IAC 22. O lodo de esgoto empregado, não higienizado com cal, foi obtido junto à Estação de Tratamento de Esgoto da SABESP, do município de Franca, SP. A caracterização química do lodo de esgoto (Quadro 2) foi realizada segundo metodologia preconizada por USEPA (1995).

O delineamento experimental utilizado foi em blocos ao acaso, constituído de sete tratamentos com três repetições, totalizando vinte e uma parcelas experimentais. Cada parcela experimental era constituída por um vaso com capacidade de 24 litros. Os tratamentos testados foram definidos pela combinação de 3 doses de calcário calcinado com 2 doses de lodo de esgoto, além de um tratamento testemunha adicional. As doses de calcário empregadas corresponderam a $0,50 \%$ e $100 \%$ da quantidade necessária para se elevar a saturação por bases a 70\%, correspondentes a 0,$0 ; 16,2$ e 32,4 g por vaso, respectivamente. As doses de lodo de esgoto corresponderam a 0 e $100 \%$ da quantidade necessária para fornecer todo o nitrogênio exigido pela cultura, ou seja, 0,0 e 94,12 g por vaso nesta ordem, de acordo com a recomendação de Raij et al. (1997), considerando a taxa de mineralização do lodo de $33,33 \%$. 
Quadro 2: Características químicas do lodo de esgoto (base seca).

Table 2: Sewage sludge chemical characteristics (dry matter base).

\begin{tabular}{|c|c|c|c|}
\hline Características & Valor & DESVPAD $^{1}$ & $\mathrm{CMP}^{2}$ \\
\hline $\mathrm{C}\left(\mathrm{g} \mathrm{kg}^{-1}\right)$ & 390,00 & 13,11 & $-*$ \\
\hline $\mathrm{pH}\left(\mathrm{H}_{2} \mathrm{O}\right)$ & 4,90 & 0,05 & - \\
\hline N-Kjeldahl $\left(\mathrm{g} \mathrm{kg}^{-1}\right)$ & 30,64 & 1,70 & - \\
\hline $\mathrm{P}\left(\mathrm{g} \mathrm{kg}^{-1}\right)$ & 9,31 & 0,49 & - \\
\hline $\mathrm{K}\left(\mathrm{mg} \mathrm{kg}^{-1}\right)$ & 37,00 & 0,52 & - \\
\hline $\mathrm{Ca}\left(\mathrm{mg} \mathrm{kg}^{-1}\right)$ & 375,0 & 8,92 & - \\
\hline $\operatorname{Mg}\left(\mathrm{mg} \mathrm{kg}^{-1}\right)$ & 45,00 & 7,00 & - \\
\hline $\mathrm{S}\left(\mathrm{g} \mathrm{kg}^{-1}\right)$ & 7,56 & 0,75 & - \\
\hline $\mathrm{Fe}\left(\mathrm{mg} \mathrm{kg}^{-1}\right)$ & 319,00 & 12,49 & - \\
\hline $\operatorname{Mn}\left(\mathrm{mg} \mathrm{kg}^{-1}\right)$ & 70,00 & 6,42 & - \\
\hline $\mathrm{Cu}\left(\mathrm{mg} \mathrm{kg}^{-1}\right)$ & 212,00 & 9,54 & 4.300 \\
\hline $\mathrm{Zn}\left(\mathrm{mg} \mathrm{kg}^{-1}\right)$ & 120,00 & 8,54 & 7.500 \\
\hline $\mathrm{Cd}\left(\mathrm{mg} \mathrm{kg}^{-1}\right)$ & 5,00 & 1,73 & 85 \\
\hline $\mathrm{Cr}\left(\mathrm{mg} \mathrm{kg}^{-1}\right)$ & 157,00 & 7,00 & - \\
\hline $\mathrm{Ni}\left(\mathrm{mg} \mathrm{kg}^{-1}\right)$ & 61,00 & 4,58 & 420 \\
\hline $\mathrm{Pb}\left(\mathrm{mg} \mathrm{kg}^{-1}\right)$ & 127,00 & 8,00 & 840 \\
\hline
\end{tabular}

Nas parcelas que não receberam lodo de esgoto, aplicou-se no plantio: $0,6 \mathrm{~g}$ de sulfato de amônio, 6,7 $\mathrm{g}$ de superfosfato simples e $1,66 \mathrm{~g}$ de cloreto de potássio e, em cobertura, 4,2 $\mathrm{g}$ de sulfato de amônio e $0,83 \mathrm{~g}$ de cloreto de potássio. As parcelas que receberam lodo de esgoto, aplicou-se no plantio e em cobertura 1,66 e 0,83 g de cloreto de potássio, respectivamente, segundo a recomendação de Raij et al. (1997), e 0,0135 g de $\mathrm{CdCl}_{2}$ (quantidade necessária de $\mathrm{Cd}$ para elevar os teores do lodo aos níveis máximos permitidos pelas normas da Cetesb (1999). 
Os tratamentos foram aplicados nos solos de cada parcela, na camada $0-10 \mathrm{~cm}$ de profundidade. Transferiu-se ou o volume correspondente de solo para saco plástico de $3 \mathrm{~kg}$ de capacidade e as frações (calcário, lodo de esgoto e fertilizantes minerais) em conformidade com os tratamentos mencionados anteriormente. Após homogeneização das frações, procedeu-se ao retorno do material aos respectivos vasos, restabelecendo o volume do solo no interior do mesmo.

Na sequiência, foram semeadas 5 sementes por vaso da cultivar IAC 22 e, uma semana após a germinação, realizou-se o desbaste mantendo-se duas plantas por vaso. A irrigação foi feita pelo método de pesagens dos vasos, mantendo a umidade correspondente a $60 \%$ da capacidade de campo de acordo com a recomendação de Klute (1986), utilizando água deionizada. As mesmas foram mantidas nos vasos por 150 dias, quando se efetuou o desmonte do experimento por meio do corte dos caules das plantas rente ao solo e separação de suas diferentes partes: raízes, parte aérea (caule, folhas e ramos) e fibra. As raízes foram separadas do solo por lavagem com água corrente.

O material obtido foi acondicionado em sacos de papel devidamente etiquetados, os quais foram encaminhados ao laboratório de análises químicas. Após lavagem das plantas em água corrente, destilada e deionizada, as mesmas foram mantidas em estufa com renovação e circulação forçada de ar $\left(60-70{ }^{\circ} \mathrm{C}\right)$ até massa constante, obtendo-se a matéria seca produzida nas diferentes partes das plantas. As mesmas foram pesadas e, em seguida, moídas em micro-moinho tipo Willey. Esse material foi submetido à digestão sulfúrica para a determinação dos teores de $\mathrm{N}$ por titulometria após destilação em microdestilador de Kjeldahl e digestão nítrico-perclórica para a determinação dos macronutrientes $(\mathrm{P}, \mathrm{K}$, $\mathrm{Ca}, \mathrm{Mg}$ e S), micronutrientes (Fe, $\mathrm{Mn}$ e $\mathrm{Zn}$ ) e metais pesados ( $\mathrm{Cd}, \mathrm{Ni}$ e $\mathrm{Pb}$ ), empregando-se colorimetria para o $\mathrm{P}$, turbidimetria para o $\mathrm{S}$, e espectrofotometria de absorção atômica para os demais elementos, conforme metodologia de Malavolta (1989), sendo os resultados expressos em relação à matéria seca de cada parte das plantas. As quantidades de nutrientes e metais pesados acumuladas nas diferentes partes da planta foram calculadas com base no peso da matéria seca de cada parte da planta e no teor de cada elemento na matéria seca.

Aos resultados obtidos, aplicou-se a análise de variância, seguindo-se da aplicação do teste de Tukey ao nível de 5\% de probabilidade para comparação das médias (Pimentel, 1990).

\section{RESULTADOS E DISCUSSÃO}

\section{Macronutrientes}

Considerando o acúmulo de N, P e K nas diferentes partes das plantas de algodoeiro (Quadro 3), verificou-se que o aumento das doses de calcário no solo resultou em menores acúmulos de $\mathrm{N}$ e $\mathrm{P}$ na parte aérea. Por outro lado, nas raízes o acúmulo de $\mathrm{P}$ e $\mathrm{K}$ foram maiores quando o calcário foi empregado. Efeito semelhante observou-se para as quantidades acumuladas de $\mathrm{N}, \mathrm{Pe}$ $\mathrm{K}$ na fibra, com o detalhe de que o aumento da dose de calcário proporcionou queda nos valores encontrados. Esses resultados, especificamente para N, são concordantes com os obtidos por Rosolem et al. (2003). As menores quantidades de $\mathrm{N}$ e $\mathrm{P}$ encontradas na parte aérea revelaram que tais elementos sofreram translocação para outras partes da planta.

O comportamento dos elementos $\mathrm{P}$ e $\mathrm{K}$ nas raízes permitem inferir que nessa parte da planta as alterações provocadas no solo foram refletidas de forma direta, o mesmo podendo se afirmar para os três elementos acumulados nas fibras. 


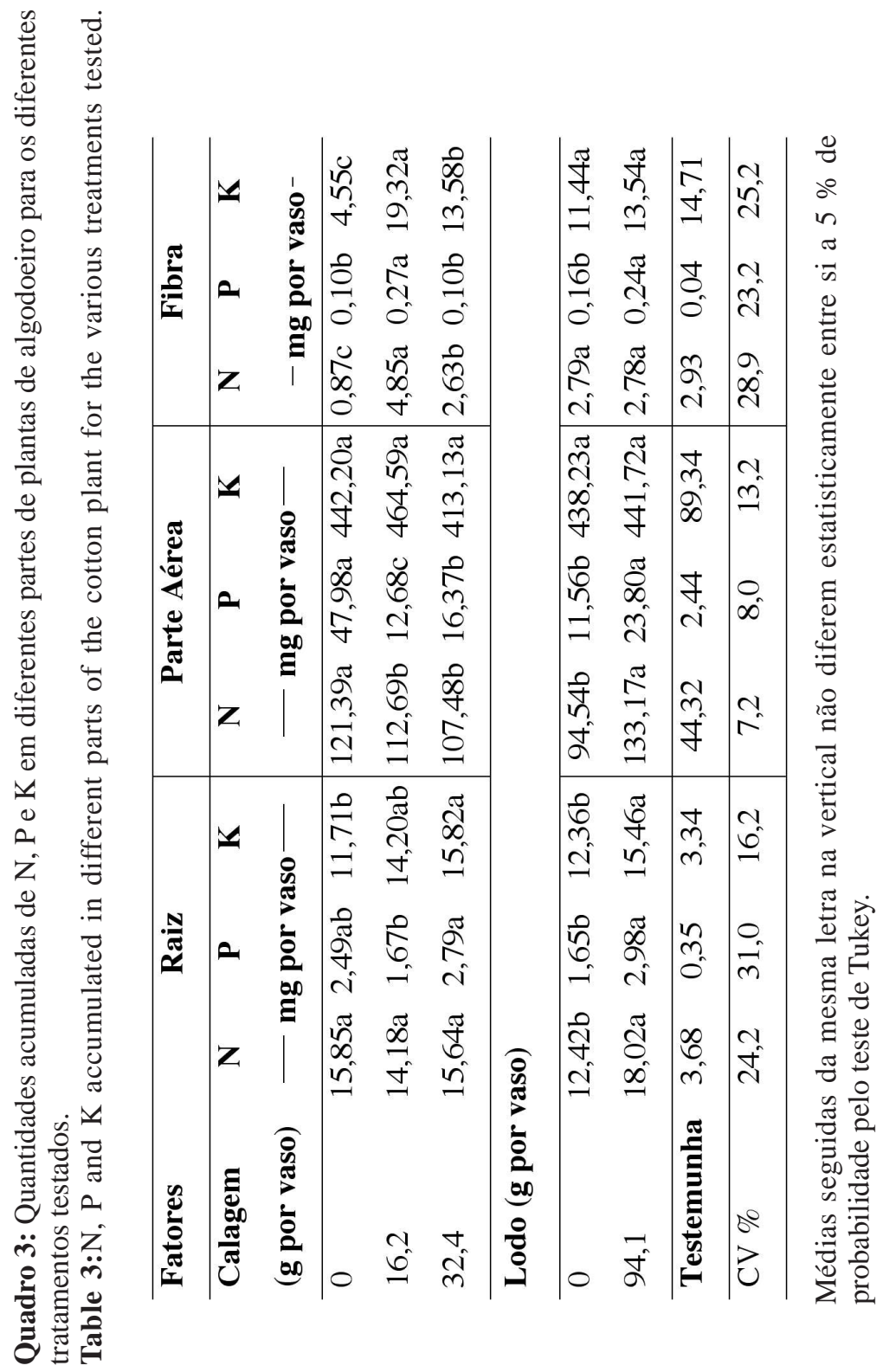


Exceto para $\mathrm{N}$ acumulado nas fibras e $\mathrm{K}$ na parte aérea e nas fibras, $\mathrm{N}, \mathrm{P}$ e $\mathrm{K}$, em todas as partes estudadas sofreram incrementos em seus acúmulos na presença de lodo de esgoto. Verificou-se ainda, que as parcelas que compunham o tratamento testemunha resultaram em plantas com menor acúmulo de $\mathrm{N}, \mathrm{P}$ e $\mathrm{K}$ do que aquelas integrantes do esquema fatorial.

Exceto para o potássio, de modo geral, verificou-se a existência de escala decrescente na ordem de grandeza dos valores acumulados (Parte aérea > Raiz > Fibra).

Considerando-se o acúmulo de $\mathrm{Ca}, \mathrm{Mg}$ e $\mathrm{S}$ nas diferentes partes de plantas de algodoeiro (Quadro 4), observou-se a adição de calcário resultou em aumento do acúmulo de $\mathrm{Ca}$ e $\mathrm{Mg}$ nas diferentes partes das plantas. Porém, o aumento da dose de calcário não proporcionou alteração. Em relação ao $\mathrm{S}$ a adição de calcário proporcionou aumento

Quadro 4: Quantidades acumuladas de $\mathrm{Ca}, \mathrm{Mg}$ e $\mathrm{S}$ em diferentes partes de plantas de algodoeiro para os diferentes tratamentos testados.

Table 4: $\mathrm{Ca}, \mathrm{Mg}$ and $\mathrm{S}$ accumulated in differents parts of cotton cotton plant for the various treatments tested.

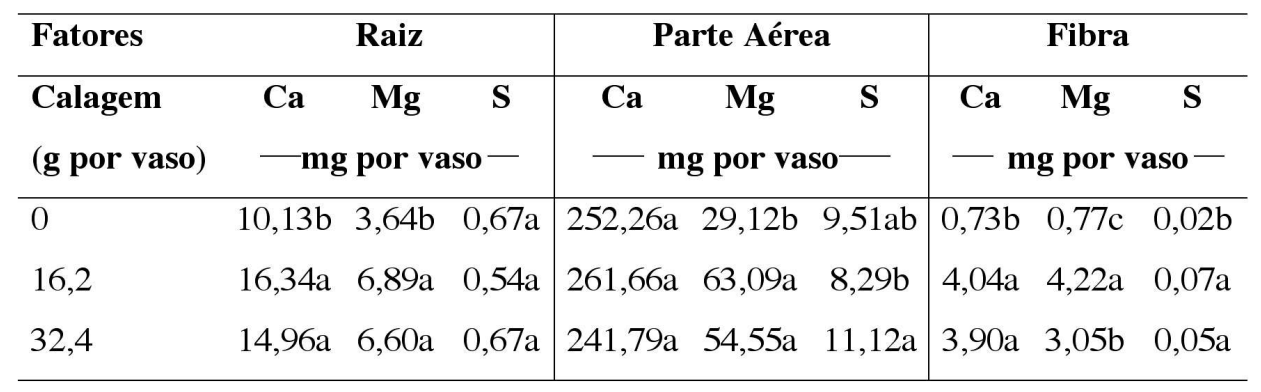

Lodo (g por vaso)

\begin{tabular}{lccc|ccc|ccc}
\hline 0 & $12,80 \mathrm{~b}$ & $4,96 \mathrm{~b}$ & $0,55 \mathrm{~b}$ & $222,19 \mathrm{~b}$ & $38,23 \mathrm{~b}$ & $8,24 \mathrm{~b}$ & $2,90 \mathrm{a}$ & $2,77 \mathrm{a}$ & $0,05 \mathrm{a}$ \\
94,1 & $14,82 \mathrm{a}$ & $6,46 \mathrm{a}$ & $0,71 \mathrm{a}$ & $281,62 \mathrm{a}$ & $59,56 \mathrm{a}$ & $11,03 \mathrm{a}$ & $2,87 \mathrm{a}$ & $2,59 \mathrm{a}$ & $0,04 \mathrm{~b}$ \\
\hline Testemunha & 3,56 & 2,21 & 0,12 & 140,99 & 22,32 & 2,86 & 3,59 & 2,16 & 0,03 \\
\hline CV \% & 12,0 & 23,4 & 20,8 & 12,9 & 21,5 & 16,5 & 27,0 & 19,1 & 23,5 \\
\hline
\end{tabular}

Médias seguidas da mesma letra na vertical não diferem estatisticamente entre si a $5 \%$ de probabilidade pelo teste de Tukey. 
nas quantidades acumuladas nas fibras. $\mathrm{Na}$ parte aérea esse efeito foi observado apenas com o emprego da maior dose.

A incorporação de lodo de esgoto no solo resultou em maior acúmulo de $\mathrm{Ca}, \mathrm{Mg}$ e $\mathrm{S}$, na parte aérea e nas raízes. Resposta semelhante foi encontrada por Simonete $e t$ al. (2003), ao avaliar o acúmulo de $\mathrm{Ca}, \mathrm{Mg}$ e $\mathrm{S}$ na parte aérea da cultura do milho, após a aplicação de doses de lodo de esgoto. Entretanto, não se verificou qualquer efeito quando se considerou o acúmulo desses elementos na fibra.

Como mencionado anteriormente, os valores de $\mathrm{Ca}, \mathrm{Mg}$ e $\mathrm{S}$ na planta do tratamento testemunha, com exceção das fibras, foram inferiores aos acúmulos ocorridos nas plantas do esquema fatorial.

\section{Micronutrientes}

Em relação ao acúmulo de Fe, Mn e Zn na parte aérea das plantas (Quadro 5), notouse que esses nutrientes acumularam-se de forma inversamente proporcional à dose de calcário, refletindo a disponibilidade desses elementos no solo influenciada pela intensidade da calagem. Resultados contrários foram obtidos por Amaral et al. (1994), quando se avaliou o acúmulo de $\mathrm{Fe}$, Mn e Zn na parte aérea da alface mediante diferentes tipos e doses de corretivos da acidez do solo. De forma contrária, os acúmulos desses micronutrientes nas raízes e nas fibras foram incrementados com a realização da calagem. Observou-se um gradiente decrescente de acúmulo na seguinte ordem: Raiz > Parte aérea > Fibra.

Quadro 5: Quantidades acumuladas de Fe, Mn e Zn em diferentes partes de algodoeiro, em função dos tratamentos testados.

Table 5: $\mathrm{Fe}, \mathrm{Mn}$ and $\mathrm{Zn}$ accumulated in cotton plant for the various treatments tested.

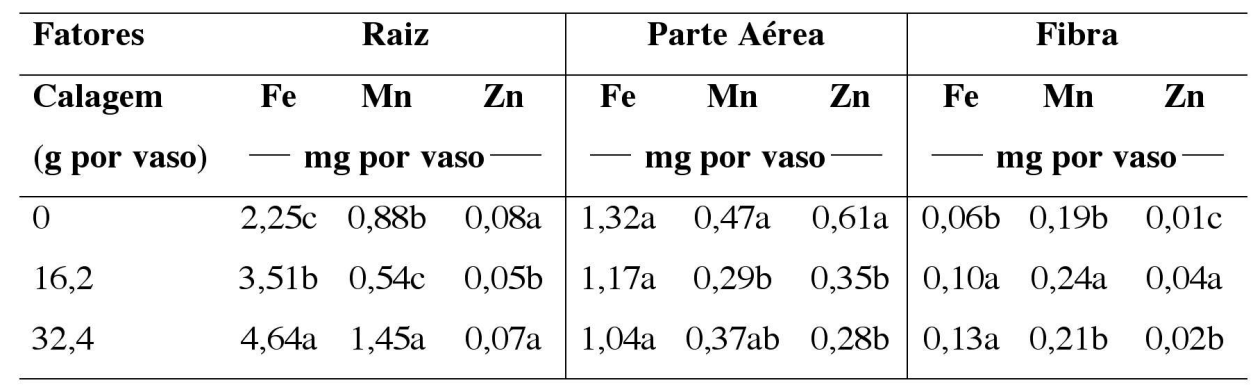

Lodo (g por vaso)

\begin{tabular}{lccc|ccc|ccc}
\hline 0 & $4,18 \mathrm{a}$ & $0,59 \mathrm{~b}$ & $0,04 \mathrm{~b}$ & $1,12 \mathrm{a}$ & $0,31 \mathrm{~b}$ & $0,29 \mathrm{~b}$ & $0,11 \mathrm{a}$ & $0,20 \mathrm{~b}$ & $0,02 \mathrm{a}$ \\
94,1 & $2,78 \mathrm{~b}$ & $1,32 \mathrm{a}$ & $0,09 \mathrm{a}$ & $1,24 \mathrm{a}$ & $0,44 \mathrm{a}$ & $0,55 \mathrm{a}$ & $0,09 \mathrm{a}$ & $0,23 \mathrm{a}$ & $0,02 \mathrm{a}$ \\
\hline Testemunha & 2,92 & 0,13 & 0,01 & 3,24 & 0,13 & 0,10 & 0,03 & 0,07 & 0,02 \\
\hline CV \% & 17,6 & 17,1 & 9,3 & 20,9 & 25,4 & 16,8 & 32,0 & 8,1 & 14,3 \\
\hline
\end{tabular}

Médias seguidas da mesma letra na vertical não diferem estatisticamente entre si a $5 \%$ de probabilidade pelo teste de Tukey. 


\section{Metais pesados}

Quanto ao acúmulo de cádmio, níquel e chumbo em diferentes partes de plantas de algodoeiro (Quadro 6), verificou-se que em todas as partes estudadas o aumento da calagem resultou em maior acúmulo de $\mathrm{Pb}$. Não foi possível apresentar os valores de $\mathrm{Cd}$ acumulados na parte aérea, uma vez que os teores presentes nos extratos analisados apresentaram valores inferiores ao limites de detecção da metodologia empregada.
Em relação ao $\mathrm{Ni}$ acumulado, independentemente da parte da planta considerada, a dose de calcário 16,02 g por vaso, resultou em maiores acúmulos deste elemento. Ainda, foi nítida a redução desse acúmulo nas parcelas em que a dose máxima de calcário foi empregada. Ressaltando a importância da correção da acidez do solo quando se pretende limitar a disponibilidade desse elemento nas plantas.

Quadro 6: Quantidades acumuladas de Cádmio, Níquel e Chumbo em diferentes partes de plantas de algodoeiro para os diferentes tratamentos testados e variáveis estatísticas. Table 6: $\mathrm{Cd}$, $\mathrm{Ni}$ and $\mathrm{Pb}$ accumulated in cotton plant for different parts, in a function of tested treatments and statistical parameters.

\begin{tabular}{|c|c|c|c|c|c|c|c|c|c|}
\hline $\begin{array}{l}\text { Fatores } \\
\text { Calagem }\end{array}$ & \multicolumn{3}{|c|}{ Raiz } & \multicolumn{3}{|c|}{ Parte Aérea } & \multicolumn{3}{|c|}{ Fibra } \\
\hline $\begin{array}{l}\text { Calagem } \\
\text { (g por vaso) }\end{array}$ & $\begin{array}{l}\mathbf{C d} \\
-\mathbf{n}\end{array}$ & $\begin{array}{l}\mathrm{Ni} \\
\text { g por va }\end{array}$ & $\begin{array}{r}\mathbf{F} \\
\text { aso- }\end{array}$ & $\begin{array}{r}\mathrm{Cd}^{1} \\
\\
\mathrm{I}\end{array}$ & $\begin{array}{c}\text { Ni } \\
\text { mg por v }\end{array}$ & $\begin{array}{r}\mathbf{P b} \\
\text { vaso } \\
\end{array}$ & $\begin{array}{l}\text { Cd } \\
\\
\end{array}$ & $\begin{array}{c}\text { Ni } \\
\text { ng por va }\end{array}$ & Pb \\
\hline 0 & $0,005 \mathrm{a}$ & $0,083 b$ & $0,043 \mathrm{c}$ & - & $0,025 \mathrm{a}$ & $0,349 b$ & $0,002 b$ & $0,005 \mathrm{c}$ & $0,012 b$ \\
\hline 16,2 & $0,005 \mathrm{a}$ & $0,162 \mathrm{a}$ & $0,058 \mathrm{~b}$ & - & $0,026 \mathrm{a}$ & $0,648 \mathrm{a}$ & $0,005 \mathrm{a}$ & $0,022 \mathrm{a}$ & $0,013 b$ \\
\hline 32,4 & $0,005 \mathrm{a}$ & $0,080 b$ & $0,089 a$ & - & $0,016 b$ & $0,518 \mathrm{a}$ & $0,005 \mathrm{a}$ & $0,016 b$ & $0,023 a$ \\
\hline
\end{tabular}

Lodo (g por vaso)

\begin{tabular}{lccc|ccc|ccc}
\hline 0 & $0,004 \mathrm{~b}$ & $0,010 \mathrm{a}$ & $0,051 \mathrm{~b}$ & - & $0,210 \mathrm{a}$ & $0,367 \mathrm{~b}$ & $0,004 \mathrm{a}$ & $0,017 \mathrm{a}$ & $0,016 \mathrm{a}$ \\
94,1 & $0,005 \mathrm{a}$ & $0,011 \mathrm{a}$ & $0,076 \mathrm{a}$ & - & $0,243 \mathrm{a}$ & $0,642 \mathrm{a}$ & $0,004 \mathrm{a}$ & $0,012 \mathrm{~b}$ & $0,015 \mathrm{a}$ \\
\hline Testemunha & 0,001 & 0,004 & 0,026 & - & 0,037 & 0,144 & 0,003 & 0,008 & 0,002 \\
\hline CV \% & 21,5 & 18,2 & 12,2 & - & 19,5 & 20,0 & 21,0 & 9,1 & 11,2 \\
\hline
\end{tabular}

Médias seguidas da mesma letra na vertical não diferem estatisticamente entre si a $5 \%$ de probabilidade pelo teste de Tukey.

${ }^{1}$ Abaixo do limite de detecção. 
Quanto aos efeitos decorrentes da aplicação de lodo de esgoto, observou-se uma elevação significativa do acúmulo de $\mathrm{Pb}$ na parte aérea e raiz e de $\mathrm{Cd}$ na raiz. $\mathrm{Na}$ fibra não se verificou qualquer influência para estes mesmos elementos, todavia houve um decréscimo significativo do acúmulo de $\mathrm{Ni}$ na planta. Esses resultados são, em parte, concordantes com Carneiro et al. (2001) que detectaram correlação direta entre os acúmulos de $\mathrm{Cd}$ e $\mathrm{Pb}$ em plantas de mostarda e gramíneas e o nível de contaminação do solo com tais elementos.

Em relação ao tratamento testemunha, os acúmulos encontrados foram, em geral, inferiores àqueles verificados nas plantas das parcelas que compõem o esquema fatorial, evidenciando que tanto o efeito da calagem quanto do lodo de esgoto, propiciaram às plantas de algodoeiro maior acúmulo de metais pesados em suas diferentes partes. Esses resultados vão ao encontro das considerações elaboradas por Kabata-Pendias e Pendias (1992) quando ressaltam que as plantas, em geral, podem acumular metais pesados em seus tecidos, evidenciando a grande habilidade de adaptação a várias condições químicas do ambiente, em especial, do solo. Destacam, ainda, que as mesmas se comportam como verdadeiros reservatórios de elementos metálicos presentes no solo, podendo chegar até os humanos e animais, o que ñao se aplica á cultura estudada nesse trabalho. Entretanto, destaca-se a potencialidade dessa cultura em atuar em processos de fitorremediação, através da hiperacumulação de metais pesados, cuja importância é ressaltada por Accioly e Siqueira (2000) e McGrath et al. (2000).

$\mathrm{Na}$ distribuição porcentual de $\mathrm{Cd}$ para as diferentes partes da planta (Figura 1), observou-se que na ausência de calcário a maior parte do elemento acumulou-se nas raízes. Entretanto, na presença de calcário a distribuição do elemento ocorreu em partes iguais entre raízes e fibras. Verificou-se também que a aplicação do lodo de esgoto contaminado com Cd não promoveu alterações na distribuição do elemento na planta. Soares et al. (2001), observou resultados contrários quando se estudou o acúmulo total de $\mathrm{Cd}$ em mudas de árvores cultivadas em solo contaminado por rejeitos de indústria de zinco. Ainda, segundo Oliveira et al. (2005), com o uso de doses crescentes de lodo de esgoto em dois tipos de solos, observaram que as raízes contribuíram para a retenção deste elemento na planta, cujos mecanismos são mencionados por Kabata-Pendias e Pendias (1992) e Mesquita (2002).

Em relação à porcentagem de $\mathrm{Ni}$ acumulada em diferentes partes de planta de algodoeiro (Figura 2), observou-se que a intensidade de calagem não influenciou a distribuição do Ni na planta, sendo que o elemento ficou, em sua maioria, retido nas raízes. Entretanto, a maior dose de calcário resultou em maior concentração do elemento nas fibras em detrimento da ocorrência do elemento na parte aérea. Também, verificouse que a aplicação de lodo contaminado com Cd não influenciou a distribuição de $\mathrm{Ni}$ nas diferentes partes da planta. Considerando-se a porcentagem de $\mathrm{Pb}$ acumulada em diferentes partes das plantas de algodoeiro (Figura 3), verificou-se que a aplicação de calcário, em doses crescentes, e do lodo de esgoto, não exercem influência na distribuição do elemento nas diferentes partes das plantas. Entretanto, mostrou-se maior porcentagem acumulada de $\mathrm{Pb}$ na parte aérea para os dois tratamentos, o que contraria as inferências de Malavolta (1994) e Berton (2000) na medida em que destacam que o $\mathrm{Pb}$ é absorvido pelas plantas, porém, fica retido nas raízes, ou seja, não sendo translocado às demais partes das plantas. Blaylock \& Huang (1999), sugerem que, o fato de muitas plantas acumularem $\mathrm{Pb}$ nas raízes, mas a sua translocação para a parte aérea ser muito baixa, pode ser devido à alta afinidade por sítios ligantes na parede celular. 


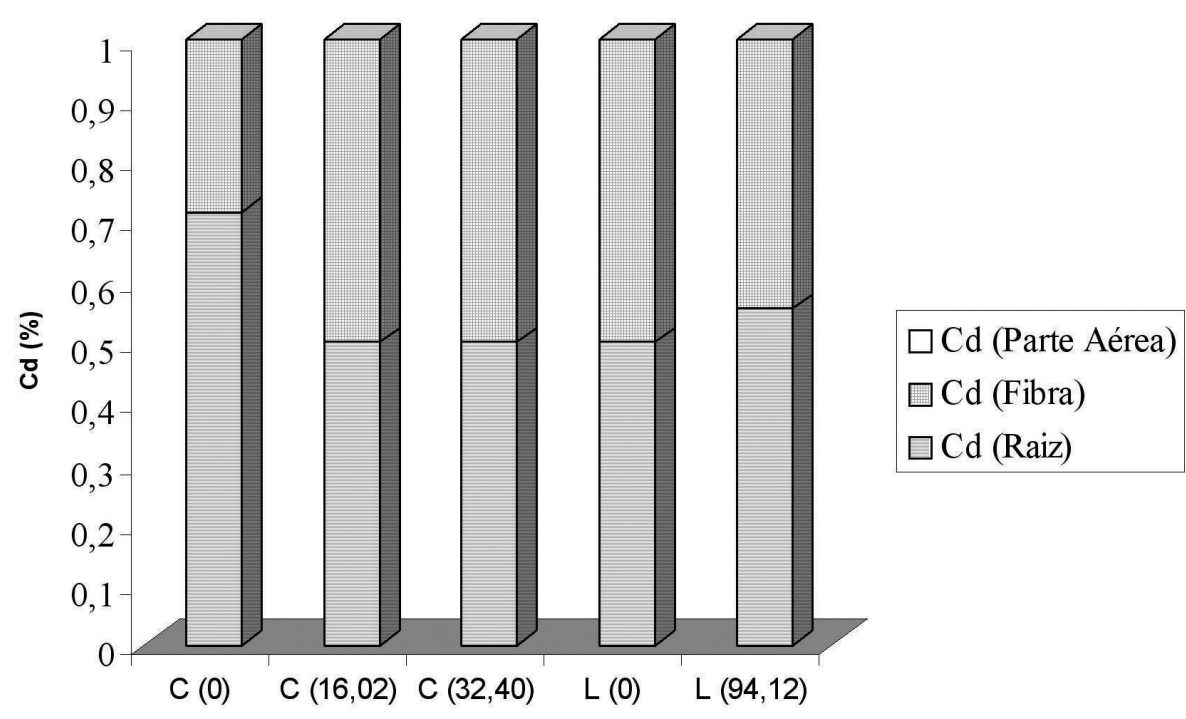

Doses calcário (C) e lodo (L) em g por vaso

Figura 1: Distribuição porcentual de Cd entre diferentes partes de planta de algodoeiro.

Figure 1: Perceptual distribution of $\mathrm{Cd}$ amongst different parts of cotton plants.

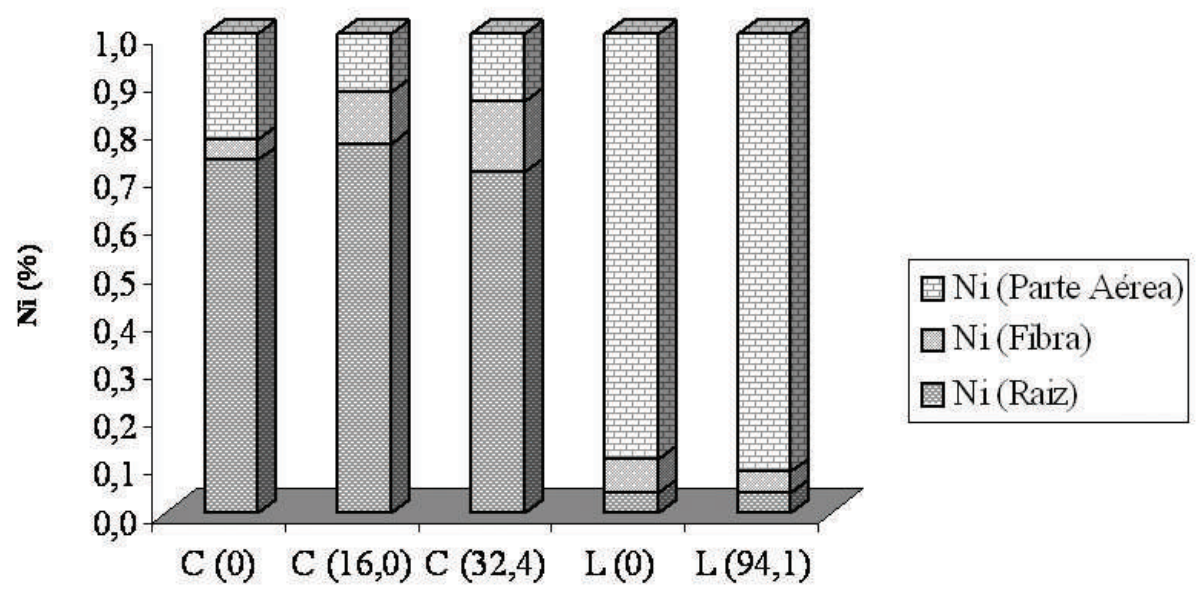

Doses calcário (C) e lodo (L) em g por vaso

Figura 2: Distribuição porcentual de Ni entre diferentes partes de planta de algodoeiro. Figure 2: Perceptual distribution of $\mathrm{Ni}$ amognst parts of cotton plants 


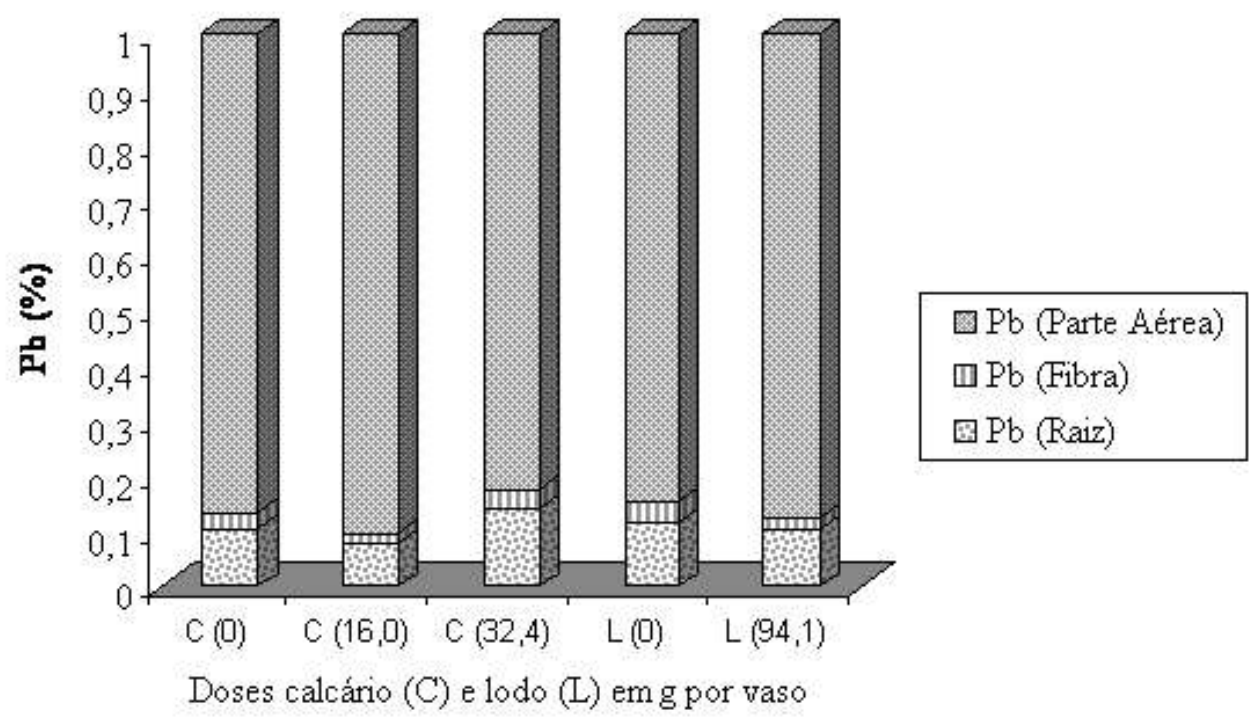

Figura 3: Distribuição porcentual de $\mathrm{Pb}$ entre diferentes partes de planta de algodoeiro. Figure 3: Perceptual distribution of $\mathrm{Pb}$ amongnst different parts of cotton plants.

\section{CONCLUSÃO}

1. Dependendo da parte da planta a ser considerada, as diferentes doses de calcário influenciaram de formas distintas o acúmulo dos macronutrientes, sendo que os maiores valores são encontrados na parte aérea. A aplicação de lodo de esgoto tendeu a elevar o acúmulo de macronutrientes nas plantas, especialmente, nas raízes e parte aérea. 2. A calagem favorece o acúmulo de Fe, $\mathrm{Mn}$ e $\mathrm{Zn}$ nas raízes e fibras. O lodo de esgoto aumenta o acúmulo de Mn e Zn na raiz, parte aérea e apenas $\mathrm{Mn}$ na fibra. Os teores de $\mathrm{Fe}$ nas raízes sofrem redução com a aplicação de lodo de esgoto.
3. Em geral, o lodo de esgoto contaminado com cádmio favoreceu o acúmulo de $\mathrm{Cd}$ e $\mathrm{Pb}$ nas raízes e parte aérea. Quando se considerou apenas a calagem, efeito semelhante foi encontrado para o $\mathrm{Cd}$ e $\mathrm{Ni}$ nas fibras.

\section{AGRADECIMENTOS}

Os autores agradecem à SABESP Franca/SP pelo fornecimento do lodo de esgoto utilizado neste experimento. 


\section{REFERÊNCIAS BIBLIOGRÁFICAS}

ACCIOLY, A.M.A.; SIQUEIRA, J.O., 2000. Contaminação química biorremediação do solo, In: R.F. NOVAES, V.H.V. ALVAREZ, C.E.G.R. SCHAEFER, (Ed). Tópicos em Ciência do Solo. Viçosa, MG: Sociedade Brasileira de Ciência do Solo, p. 299-352.

AMARAL, A.S., DEFELIPO, B.V., COSTA, L.M., FONTES, M.P.F. 1994. Liberaçãode Zn, Fe, Mn e Cd de quatro corretivos da acidez e absorção por alface em dois solos. Pesq. Agropec. Bras.Brasília, 29 (9) 1351-1358.

BERTON, R.S., 2000. Riscos de contaminação do agroecossistema com metais pesados. In: W. BETTIOL, O.A. CAMARGO, (Ed.). Impacto Ambiental do Uso Agrícola de Resíduo de Esgoto. Jaguariúna: Embrapa Meio Ambiente, p. 259268.

BLAYLOCK, M.J, HUANG, J.W., 1999. Phytoextraction of metals. In: I. RASKIN,B.D. ENSLEY, eds. Phytoremediation or toxic metals: Using plants to clean up the environment. New York, John Wiley \& Sons, p. 53-70.

CARNEIRO, M.A.C., SIQUEIRA, J.O., MOREIRA, F.M.S. 2001. Estabelecimento de plantas herbáceas em solo com contaminação de metais pesados einoculação de fungos micorrízicos arbusculares. Pesq. Agropec. Bras., Brasília, 36 (12) 1443-1452.

COMPANHIA DE TECNOLOGIA DE SANEAMENTO AMBIENTAL CETESB.,1999. Aplicação de lodos de sistemas de tratamento biológico em áreas agrícolas-Critérios para projeto e operação. São Paulo, 32 p.
EMPRESA BRASILEIRA DE PESQUISA AGROPECUÁRIA - EMBRAPA., 1999. Centro Nacional de Pesquisa de Solos. Sistema Brasileiro de Classificadão de Solos. Brasília, $412 \mathrm{p}$.

KABATA - PENDIAS, A.; PENDIAS, H ., 1992. Trace Elements in Soils and Plants. Flórida: CRC Press, 365 p.

KLUTE, A., 1986. Water retention: laboratory methods. In: A. KLUTE, (Ed.) Methods of soil analysis. Madison: American Society of Agronomy, p. 563 - 596

LOPES, J.C., RIBEIRO, L.G., ARAÚJO, M.G., BERALDO, M.R.B.S. 2005. Produção de alface com doses de lodo de esgoto. Hortic. Bras., 23 (1) 143-147.

MALAVOLTA, E., VitTi. G.C., OLIVEIRA, S.A., 1989. Avaliação do estado nutricional das plantas princípios e aplicações. Piracicaba, Potafos. 201 p.

MALAVOLTA, E., 1994. Fertilizantes e seu impacto ambiental: micronutrientes e metais pesados, mitos, mistificação e fatos.São Paulo: Produquímica, $153 \mathrm{p}$.

McGRATH, S.P., DUNHAM, S.J., CORREL, R.L., 2000. Potential for phytoextraction of zinc and cadmium from soils using hyperaccumulator plants. In: N. Terry, G. Banuelos, eds. Phytoremediation of contaminated soil and water. Boca Raton, Lewis Puclishers, p. 109-128. MESQUITA, A.A., 2002. Remediação de Remediação de áreas contaminadas por metais pesados provenientes de lodo de esgoto. Dissertação Mestrado em Ciência do Solo - Universidade Federal Rural do Rio de Janeiro, Seropédica, $68 \mathrm{p}$ 
NASCIMENTO, C.W.A., BARROS, D.A.S., MELO, E.E.C., OLIVEIRA, A.B. 2004. Alterações químicas em solos e crescimento de milho e feijoeiro após aplicação de lodo de esgoto. Rev. Bras. Cienc. Solo, Viçosa, 28 (2) 385-392.

NRIAGU, J.O., PACYNA, J.M. 1988. Quantitative assessment of worldwide contamination of air, water and soils with trace metals. Nature, London, 333 (6169) 134-139.

OLIVEIRA, C., AMARAL SOBRINHO, N.M.B., MARQUES, V.S., MAZUR, N. 2005. Efeitos da aplicação do lodo de esgoto enriquecido com cádmio e zinco na cultura do arroz. Rev. Bras. Cienc. Solo, Viçosa, 29 (1) 109-116.

PEDROZA, J.P., HAANDEL, A.C. van., BELTRÂO, N.E.M., DIONÍSIO, J.A. 2003. Produção e componentes do algodoeiro herbáceo em função da aplicação de biossólidos. Rev. Bras. Eng. Agríc. e Amb., Campina Grande, 7 (3) 483-488.

PIMENTEL GOMES, F., 1990. Curso de estatística experimental. 13 ed. Piracicaba: Nobel, 468 p.

RAIJ, B. van, QUAGGIO, J.A., CANTARELA, H., FERREIRA, M.E., LOPES, A.S. BATAGLIA, O.C., 1987. Análise química do solo para fins de fertilidade. Campinas, Fundação Cargill, 170 p.

RAIJ, B. van, CANTARELLA, H., QUAGGIO, J.A., FURLANI, A.M.C., 1997. Recomendações de adubação e calagem para o Estado de São Paulo. $2^{\text {a }}$ ed. rev.atual. Campinas: Instituto Agronômico, 285 p. (Boletim Técnico 100).
ROCHA, R.E.M.; PIMENTEL M.S.; ZAGO, V.C.P.; RUMJANEK, N.G.; POLLI H. 2003. Avaliação de biossólido de águas servidas domiciliares como adubo em couve. Pesq. Agropec. Bras., Brasília, 38 (12) 1435-1441.

ROSOLEM, C.A., FOLONI, J.S.S., OLIVEIRA, R.H. 2003. Dinâmica do nitrogênio no solo em razão da calagem e adubação nitrogenada, com palha na superfície. Pesq. Agropec. Bras., Brasília, 38 (2) 301-309.

SIMONETE, M.A., KIEHL, J.C., ANDRADE, C.A., TEIXEIRA, C.F.A. 2003. Efeito do lodo de esgoto em um argissolo e no crescimento e nutrição de milho. Pesq. Agropec. Bras., Brasília, 38 (10) 1187-1195.

SOARES, C.R.F.S., ACCIOLY, A.M.A., MARQUES, T.C.L.L.S.M., SIQUEIRA, J.O., MOREIRA, F.M..S. 2001. Acúmulo e distribuição de metais pesados nas raízes, caule e folhas de mudas de árvores em solo contaminado por rejeitos de indústria de zinco, Rev. Bras. Fisiol. Veg., Lavras, 13 (3) 302-315.

UNITED STATES ENVIRONMENTAL PROTECTION AGENCY USEPA, 1995. EPA/832-B-93-005. A guide to the biosolids risk assessments for the EPA Part 503 rule. Fed. Reg. 143p. 